\title{
Montelukast Prevents Mice Against Acetaminophen-Induced Liver Injury
}

\author{
Shiyun $\mathrm{Pu}^{1}$, Qinhui Liu' ${ }^{2}$, Yanping $\mathrm{Li}^{2}$, Rui Li ${ }^{1}$, Tong $W u^{1}$, Zijing Zhang ${ }^{3}$, Cuiyuan Huang ${ }^{1}$, \\ Xuping Yang ${ }^{1}$ and Jinhan $\mathrm{He}^{1,2 *}$
}

\begin{abstract}
Department of Pharmacy, West China Hospital of Sichuan University and Collaborative Innovation Center of Biotherapy, Chengdu, China, ${ }^{2}$ Laboratory of Clinical Pharmacy and Adverse Drug Reaction, West China Hospital of Sichuan University and Collaborative Innovation Center of Biotherapy, Chengdu, China, ${ }^{3}$ Molecular Medicine Research Center, State Key Laboratory of Biotherapy and Cancer Center, West China Hospital of Sichuan University and Collaborative Innovation Center of Biotherapy, Chengdu, China
\end{abstract}

\section{OPEN ACCESS}

Edited by: Ralf Weiskirchen, RWTH Aachen University, Germany

Reviewed by: Parames C. Sil, Bose Institute,

India

Mohammad Ibrahim AIWahsh, University of Heidelberg, Germany

Weidong Shen, Jiangyin People's Hospital, China

Heiko Mühl, Goethe University Frankfurt, Germany

*Correspondence: Jinhan $\mathrm{He}$ jinhanhe@scu.edu.cn

Specialty section: This article was submitted to Gastrointestinal and Hepatic Pharmacology,

a section of the journal Frontiers in Pharmacology

Received: 10 April 2019 Accepted: 22 August 2019 Published: 18 September 2019

Citation:

PU S, LiU Q, Li Y, LiR, WU T, Zhang $Z$, Huang $C$, Yang $X$ and $\mathrm{He} J$ (2019) Montelukast Prevents Mice Against Acetaminophen-Induced Liver Injury.

Front. Pharmacol. 10:1070. doi: 10.3389/fphar.2019.01070
Acetaminophen (APAP) is a widely used over-the-counter antipyretic and analgesic drug. Overdose of APAP is the leading cause of hospital admission for acute liver failure. Montelukast is an antagonist of cysteinylleukotrienereceptor 1 (Cysltr1), which protects from inflammation and oxidative stress. However, the function of montelukast in APAP-induced hepatotoxicity remains unknown. In this study, we examined whether pharmacological inhibition of Cystlr1 could protect mice against APAP-induced hepatic damage. We found that APAP treatment upregulated messenger RNA and protein levels of Cysltr1 both in vitro and in vivo. Pharmacological inhibition of Cysltr1 by montelukast ameliorated APAPinduced acute liver failure. The hepatoprotective effect of montelukast was associated with upregulation of hepatic glutathione/glutathione disulfide level, reduction in c-Jun$\mathrm{NH} 2$-terminal kinase activation and oxidative stress. In mouse primary hepatocytes, inhibition of Cysltr1 by montelukast ameliorated the expression of inflammatory-related genes and APAP-induced cytotoxicity. We conclude that montelukast may be used to treat APAP-induced acute hepatic injury.

Keywords: montelukast, acetaminophen, cysteinyl leukotriene receptor 1, glutathione, JNK

\section{INTRODUCTION}

Acetaminophen (APAP) is a widely used over-the-counter antipyretic and analgesic drug (Lee, 2017). Although it was considered as a safe drug, APAP overdosage can lead to hepatocellular necrosis and acute liver injury (Nakagawa et al., 2008; Lee, 2017).

The majority of APAP is metabolized by conjugating enzymes UDP-glucuronosyltransferase (UGT) and sulfotransferase (SULT) in the liver to nontoxic compounds, followed by renal and biliary excretion (Yan et al., 2018). Less than 10\% of APAP is bioactivated by phase I cytochrome P450 (CYP) enzymes such as CYP1A2, CYP2E1, and CYP3A4 into reactive intermediate metabolite N-acetyl-pbenzoquinone-imine (NAPQI), which causes acute liver injury (Thummel et al., 1993; Shayiq et al., 1999). NAPQI is detoxified by glutathione (GSH) to form a hazard-free metabolite (Basavarajaiah et al., 2004). With APAP overdose, GSH is depleted, and as a result, NAPQI accumulates and binds to proteins and cause oxidative stress and the activation of c-Jun- $\mathrm{NH}_{2}$-terminal kinase (JNK) in

Abbreviations: APAP, acetaminophen; MK, montelukast; Cysltr1, Cysteinyl leukotriene receptor 1; JNK, c-Jun-NH2-terminal kinase; NAPQI, N-acetyl-p-benzoquinone-imine; 5-LO, 5-lipoxygenase. 
the liver (Nakagawa et al., 2008). JNK activation could trigger inflammation and recruitment of monocytes and neutrophils (Woolbright and Jaeschke, 2017).

The 5-lipoxygenase (5-LO) pathway plays a significant role in the pathophysiology of APAP-induced liver inflammation and injury (Hohmann et al., 2013; Pu et al., 2016). Overdose of APAP was thought to upregulate 5-LO (Suciu et al., 2016), resulting in increasing cysteinyl leukotrienes (CysLTs) secretion (Hohmann et al., 2013). Cysteinyl leukotriene receptor 1 (Cysltr1), a receptor for CysLTs, mediated various disease in humans as well as animal models. Previous reports indicated that the expression of Cysltr1 is upregulated in adenoid hypertrophy (Gao et al., 2018). Activation of Cysltr1 stimulates JNK phosphorylation (Lei et al., 2019), leading to the trigger of inflammation (Kondeti et al., 2016). Knockout of Cysltr1 prevents mice from irritantinduced asthma (McGovern et al., 2016) and also reduces colitisassociated colon cancer (Osman et al., 2017).

Montelukast, as a selective inhibitor of Cysltr1, is clinically used for the prevention and long-term treatment of asthma (Lynch et al., 1999). Several studies investigated that montelukast has an antioxidant effect in intestinal ischemia-reperfusion injury ( $\mathrm{Wu}$ et al., 2015) and also reduces cardiac damage (Khodir et al., 2016). The beneficial effects of montelukast have also been reported in various experimental models of liver injury (El-Boghdady et al., 2017; Hegab et al., 2018). However, the mechanism of montelukast in APAP-induced hepatotoxicity remains unknown. Under the light of this information, we investigated whether the pharmacological inhibition of Cysltr1 by montelukast in mice could protect against APAP-induced hepatotoxicity.

\section{MATERIALS AND METHODS}

\section{Chemicals and Reagents}

APAP and dimethylsulfoxide (DMSO) was purchased from Sigma-Aldrich (St. Louis, USA). Montelukast and zafirlukast were purchased from meilunbio (Dalian, China). GSH/ glutathionedisulfide(GSSG)assaykit was obtained from the Nanjing Jiancheng Bioengineering Institute (Nanjing, China). The lactate dehydrogenase (LDH) cytotoxicity assay kit and the mitochondrial membrane potential assay kit with JC-1 were offered by the Beyotime Institute of Biotechnology (Shanghai, China). Primary antibodies according Cysltr 1 and $\beta$-actin were purchased from Abclonal (Wuhan, China), and the secondary antibody was purchased from Jackson ImmunoResearch (PA, USA).

\section{Animals}

This study used 8-week-old C57BL/6J mice (22-25 g), which were randomly selected for this experimental study. The acute hepatic injury was induced by oral administration of APAP $(200 \mathrm{mg} / \mathrm{kg}$ ) before $16 \mathrm{~h}$ fasting as described (Saini et al., 2011; $\mathrm{Pu}$ et al., 2016). For therapeutic experiment, a dose of $3 \mathrm{mg} / \mathrm{kg}$ (Hamamoto et al., 2017) of montelukast was prepared in a $0.5 \%$ carboxy methyl cellulose. Mice were gavaged in a volume of 100 $\mu \mathrm{l}$ at $1 \mathrm{~h}$ after APAP administration. Mice were killed by $\mathrm{CO}_{2}$ at $12 \mathrm{~h}$ after APAP administration, and blood and liver tissue were harvested for histology. All mice in experiment were housed at West China Hospital, Sichuan University in accordance with the guidelines of the animal care utilization committee of the institute. Food and water were made freely available to the mice, except where otherwise state.

\section{Serum Isolation and Alanine Transaminase and Aspartate Aminotransferase Detection}

Blood was placed at $4^{\circ} \mathrm{C}$ for $1 \mathrm{~h}$, then centrifuged at $3,000 \times g$ for $10 \mathrm{~min}$. The serum was collected from the supernatant and was separately preserved at $-80^{\circ} \mathrm{C}$. Serum alanine transaminase (ALT) and aspartate aminotransferase (AST) levels were detected using commercial assay kits (BioSino Bio-Technology \& Science Inc.).

\section{H\&E Staining}

The smallest lobe of the livers was removed and immediately fixed in 10\% neutral-buffered formalin and embedded in paraffin, sectioned at $4 \mu \mathrm{m}$. For H\&E staining, liver sections were stained with hematoxylin and eosin. Samples were visualized under a light microscope (Nikon, Japan).

\section{Hepatic GSH/GSSG Detection}

Mice were killed at different time points after administration of APAP. Livers were isolated and immediately removed surface blood in saline, then homogenized in 5\% trichloroacetic acid, then centrifuged at 3,500 rpm for $10 \mathrm{~min}$. The supernatant was used to detect liver GSH/GSSG level by hepatic GSH/GSSG assay kit (Nanjing Jiancheng Bioengineering Institute, China).

\section{Detection of Liver $\mathrm{H}_{2} \mathrm{O}_{2}$ Level and Thiobarbituric Acid Reactive Substances Production}

$\mathrm{H}_{2} \mathrm{O}_{2}$ level and thiobarbituric acid reactive substances (TBARS) in liver were measured as described (Pu et al., 2016).

\section{Isolation and Treatment of Primary Mouse Hepatocytes}

Hepatocytes were isolated from 6-week-old C57 BL/6J mice and cultured as described (Kizu et al., 2015; Furuta et al., 2016). Montelukast was dissolved in DMSO, and DMSO was used a control. APAP was dissolved in high-glucose Dulbecco's modified Eagle's medium, which was supplemented with $2 \%$ fetal bovine serum. For therapeutic experiment, primary hepatocytes were pretreated with montelukast $(1,5$, and $10 \mu \mathrm{M})$ or vehicle (0.02\% DMSO) $1 \mathrm{~h}$ before APAP $(2.5 \mathrm{mM})$ administration (Furuta et al., 2016).

\section{Cell Death}

Cell death was measured using the $\mathrm{LDH}$ cytotoxicity assay kit (Beyotime, China) and the mitochondrial membrane potential 
assay kit (Beyotime, China) according to the manufacturers' recommendations. For the LDH release detection, Triton X-100, $1 \%(w / v)$, was used as a positive control. The percentage of $\mathrm{LDH}$ released was calculated as a ratio of the positive control $\mathrm{LDH}$ released (Furuta et al., 2016). For the mitochondrial membrane potential detection, the cells were incubated with $5 \mathrm{mg} / \mathrm{L}$ JC- 1 dye for $30 \mathrm{~min}$ at $37^{\circ} \mathrm{C}$ in the dark and washed twice with the dye buffer. Then, the cells were quickly subjected to a fluorescence microscope for captured red or green fluorescence (Chen et al., 2018).

\section{RT-PCR Analysis}

Total liver RNA and cellular RNA were extracted using the Trizol Reagent (Invitrogen, Carlsbad, CA), and 1,000 ng of RNA was reverse transcribed to complementary DNA using a PrimeScript RT reagent Kit (Takara RR037A). Quantitative, real-time PCR was performed on the CFX96 real-time system (Biorad) using either SYBR Green Mix (Biorad). All of the primers used with SYBR green were designed to span at least one exon to minimize the possibility of nonspecific amplification from the genomic DNA. The expression of $18 \mathrm{~s}$ gene was used as a housekeeping gene to normalize data. Specific primer sequences are in Supplementary Table 1. Relative messenger RNA (mRNA) expression was quantified using the comparative $\mathrm{CT}(\mathrm{Ct})$ method and expressed as $2^{\wedge}(-\Delta \Delta \mathrm{Ct})$. Amplification specificity was evaluated by determining the product melting curve. Results are expressed as indicated in the figure legends. The following program was used: one step at $95^{\circ} \mathrm{C}$ for $2 \mathrm{~min}, 40$ cycles of denaturation at $95^{\circ} \mathrm{C}$ for $30 \mathrm{~s}$, and annealing and elongation at $60^{\circ} \mathrm{C}$ for $45 \mathrm{~s}$.

\section{Western Blotting}

Western blotting analyses were performed with protein extracts from liver homogenates $(50 \mu \mathrm{g})$ using anti-p-ERK (1:2,000 dilution, Santa Cruz), anti-ERK (1:2,000 dilution, Santa Cruz), anti-p-JNK (1:1,000 dilution, CST), and anti-JNK (1:1,000 dilution, CST) antibodies. Immunoreactive bands were visualized on nitrocellulose membranes using alkaline phosphatase-conjugated antimouse or rabbit antibody and the Odyssey detection system (LI-COR, USA).

\section{Statistical Analysis}

Experiments were repeated at least three times with similar results. Quantitative results are expressed as the mean \pm SEM. Statistical significance was determined by Student's unpaired two-tailed $t$ test or one-way ANOVA multiple comparison test. $P<0.05$ was considered statistically significant.

\section{RESULTS}

\section{APAP Induced Cysltr1 Expression Both In Vivo and In Vitro}

To examined whether APAP affected the expression of Cysltr1, C57 BL/6J mice underwent gavage with saline or APAP (200 mg/ $\mathrm{kg}$ ) after $16 \mathrm{~h}$ fasting. As expected, compared to saline treatment, acute APAP treatment induced liver damage (Figures 1A, B).
The mRNA and protein levels of Cysltr1 were significantly upregulated in APAP-treated mice liver compared with vehicle group (Figures $\mathbf{1 C}-\mathbf{E})$. In contrast, Blt1 $\left(\mathrm{LTB}_{4}\right.$ receptor 1$)$ was slightly decreased after APAP treatment (Supplementary Figure 1). APAP did not affect the expression of other leukotriene receptors such as Blt2 and Cysltr2 (Supplementary Figure 1).

We then isolated primary hepatocytes from C57/BL6J mice and assessed the mRNA and protein levels of Cysltr1 after APAP administration in vitro. Similarly, the mRNA and protein level of Cysltr1 were increased in APAP-treated hepatocytes compared with the vehicle group (Supplementary Figures 2A, C, D). However, the expression of Blt1, Blt2, and Cysltr2 did not change after APAP administration (Supplementary Figures 2A, B).

\section{Pharmacological Inhibition of Cysltr1 Prevented Acetaminophen-Induced Liver Injury}

The increased expression of Cysltr1 in APAP overdose-treated mouse liver prompted us to determine whether pharmacological inhibition of Cysltr1 would affect APAP-induced liver toxicity. C57BL/6J mice were treated with vehicle or the Cystlr1 antagonist, montelukast ( $3 \mathrm{mg} / \mathrm{kg}), 1 \mathrm{~h}$ after saline or APAP administration (Figure 2A). Mice were killed $12 \mathrm{~h}$ after saline or APAP treatment, and blood and liver tissues were harvested. Montelukast treatment significantly decreased serum levels of ALT and AST (Figures 2B, C) and alleviated liver damage as indicated by $\mathrm{H} \& \mathrm{E}$ staining in APAP-treated groups (Figures 2D, E). Montelukast has no effect on saline-treated groups (Figures 2B-E). These results suggested that inhibition of Cysltr1 protected mice from APAP-induced liver injury.

\section{Montelukast Treatment Did Not Alter APAP Phase I/II Enzymes and Hepatic Transporters}

Resistance to APAP toxicity in montelukast-treated mice led us to speculate that pharmacological inhibition of Cysltr1 may alter the formation of toxicity-induced metabolites and/or promote APAP clearance. To investigate whether montelukast administration affected APAP metabolism, we first measured phase I enzymes, which are responsible for the formation of toxic APAP metabolites. However, the expression of Cyp1a2, $C y p 2 e 1$, and Cyp3a11 in livers were nearly comparable between montelukast-treated and vehicle group (Supplementary Figures 3A-C). Phase II enzymes were known to detoxify APAP. However, the expression of UDP-glucuronosyltransferase 1A1 (Ugt1a1) and sulfotransferase 2A1 (Sult2a1) did not change in montelukast-treated mice (Supplementary Figures 3D, E). Transporters were also associated with APAP-induced acute liver damage. However, montelukast administration did not alter the expression of transporters (Supplementary Figures 4A-C).

\section{Montelukast Treatment Upregulated Hepatic GSH Level and Gst $\alpha 2$ Expression.}

GSH plays a critical role in detoxifying the reactive intermediate of APAP (Nakagawa et al., 2008). With overdoses of APAP 
A

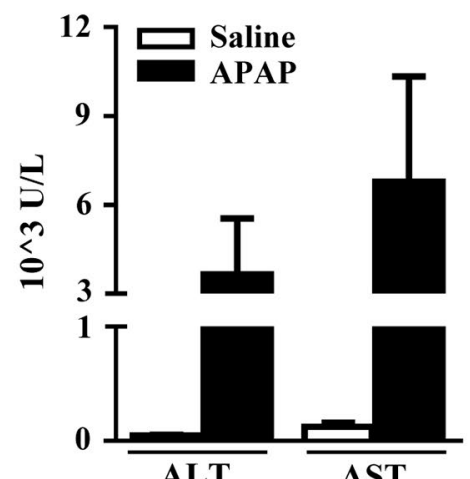

B

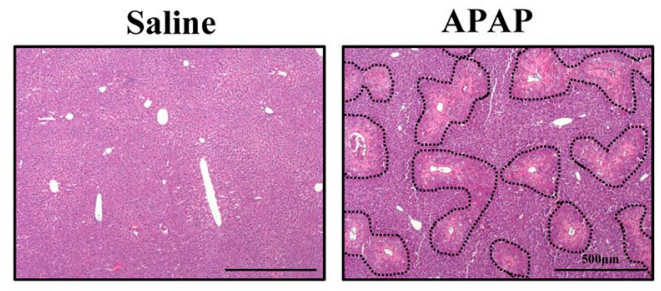

C

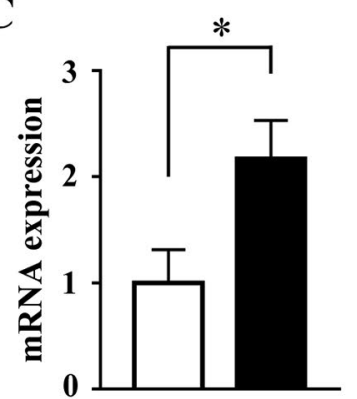

D

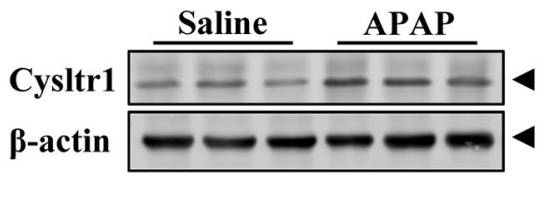

$\mathbf{E}$

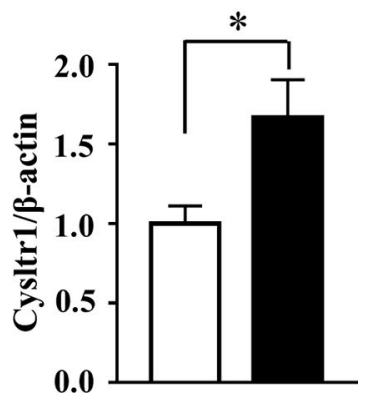

FIGURE 1 | Acute acetaminophen (APAP) treatment upregulated Cys/tr1 expression in vivo. C57BL/6J mice fasted overnight, then were orally administered with saline or APAP (200 mg $/ \mathrm{kg}$ ) for $12 \mathrm{~h}$. Data are mean \pm SEM, $n=5$ for saline group, $n=6$ for APAP group. (A) Detection of serum alanine transaminase (ALT) and aspartate aminotransferase (AST). (B) H\&E staining for livers from saline or APAP-treated mice. APAP-induced centrilobular necrosis was indicated by dotted line. (C) Real-time PCR analysis of hepatic messenger RNA (mRNA) expression of Cysltr1. (D) Western blot analysis of Cysltr1. (E) Quantification of Cysltr1 to $\beta$-actin. Data are mean \pm SEM, $n=5,{ }^{*} p<0.05$.

administration, hepatic GSH/GSSG level was markedly depleted in the vehicle group at $3 \mathrm{~h}$ and recovered at $12 \mathrm{~h}$ (Figure 3A). However, hepatic GSH/GSSG level was higher in montelukast-treated mice at $3 \mathrm{~h}$ (Figure $\mathbf{3 A}$ ). There was no difference in hepatic GSH/GSSG levels between vehicle and montelukast-treated groups at $12 \mathrm{~h}$ after APAP administration. Glutathionylation is catalyzed by a group of enzymes called the glutathione S-transferase (GST). We found enhanced Gsta2 expression in montelukast treated liver (Figure 3B). However, the mRNA levels of GSTa1, GSTm1, and GSTm2 were unaffected (Supplementary Figures 4D-F).

\section{Montelukast Decreased ROS Levels in Mice}

Hepatic GSH depletion accumulates APAP metabolites covalently bound to protein, which cause oxidative stress and lipid peroxidation (Nakagawa et al., 2008). We evaluated the $\mathrm{H}_{2} \mathrm{O}_{2}$ and TBARS level in mice treated with saline or APAP. We found that APAP greatly increased hepatic $\mathrm{H}_{2} \mathrm{O}_{2}$ and TBARS level in both vehicle and montelukast-treated mice (Figures 3C, D). However, montelukast decreased both hepatic $\mathrm{H}_{2} \mathrm{O}_{2}$ and TBARS level compared with vehicle-treated mice (Figures 3C, D).

\section{Pharmacological Inhibition of Cysltr1 by Montelukast Prevented Acetaminophen- Induced Liver Inflammation and JNK Activation}

With overdoses of APAP, hepatic GSH depletion resulted in APAP metabolites covalently bound to protein, which further exacerbates hepatic toxicity by causing inflammatory responses (Luster et al., 2000). In our study, levels of the inflammatory cytokines, including Mcp-1, TNF- $\alpha, I l-6$, and $I l-18$, were increased by APAP administration as expected (Figures 4A-D). Montelukast administration alleviated APAP-induced inflammation (Figures 4A-D). The phosphorylation of JNK1/2 and ERK1/2, mediators of inflammation, were increased by APAP overdosage (Arthur and Ley, 2013). Montelukast suppressed the phosphorylation levels of JNK1/2 after APAP administration (Figures 4E, F). Meanwhile, pharmacological inhibition of Cysltr1 slightly inhibited ERK1/2 activation in APAP-treated groups (Figures 4E, G).

\section{Pharmacological Inhibition of Cysltr1 Protects Mouse Primary Hepatocytes From APAP-Induced Cell Death.}

To further confirm the protective role of montelukast in APAPinduced hepatotoxicity, primary hepatocytes were isolated and 
A

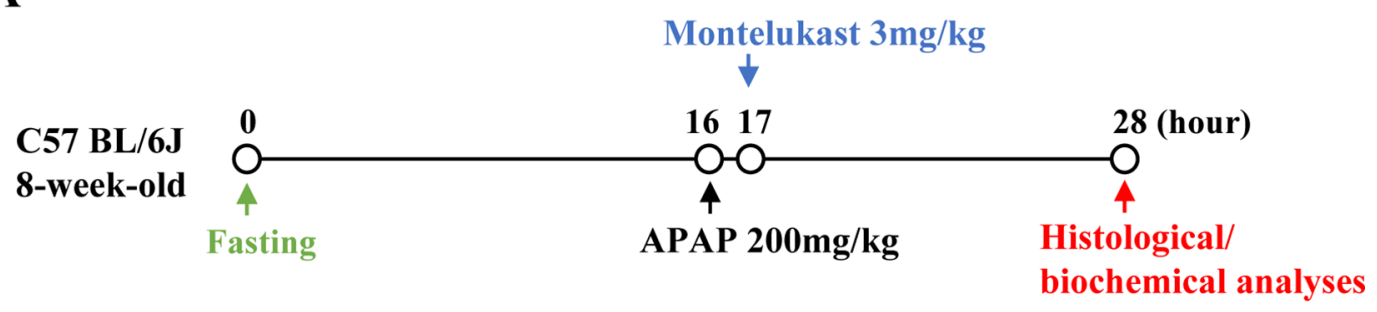

B

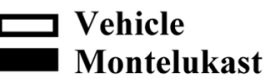

C

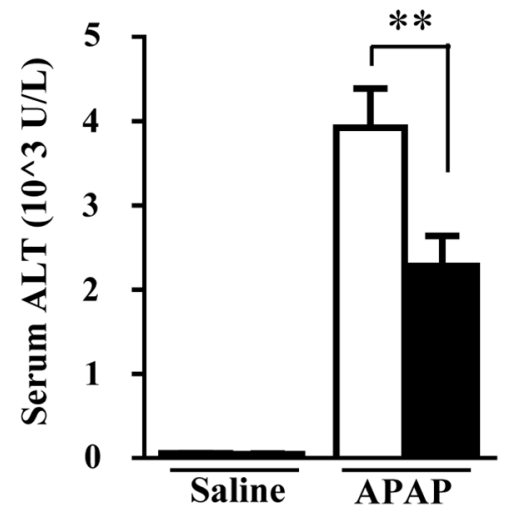

D

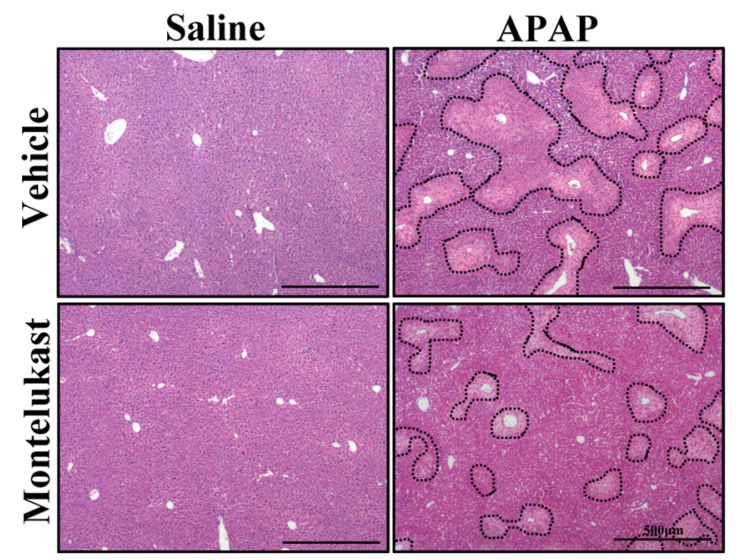

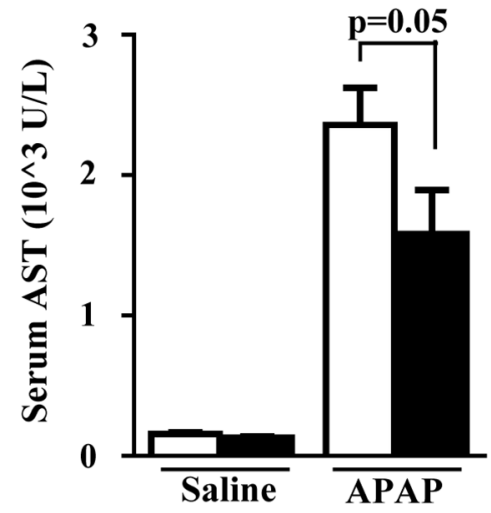

E

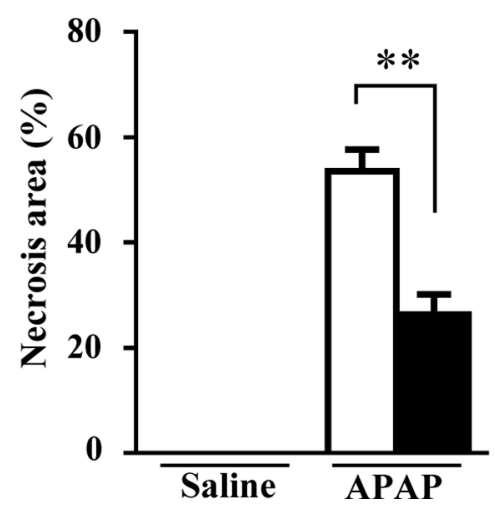

FIGURE 2 | Pharmacological inhibition of Cysltr1 protected against APAP-induced hepatotoxicity. (A). Schedule of montelukast administration in APAP-overdose mice. Montelukast (3 mg/kg) or vehicle were administered $1 \mathrm{~h}$ after APAP treatment. At $12 \mathrm{~h}$ after APAP administration, mice were killed, and blood and liver tissues were collected. Serum levels of ALT (B) and AST (C). (D) H\&E staining of liver sections from APAP- or saline-treated mice. APAP-induced centrilobular necrosis was indicated by dotted line. (E) Quantification of liver necrosis area. Data are mean \pm SEM, $n=5$ for saline groups, $n=7$ for APAP groups, ${ }^{\star \star} p<0.01$.

pretreated with montelukast $1 \mathrm{~h}$ before APAP administration. APAP treatment remarkably increased cytotoxicity as expected (Figures 5A, B). Montelukast decreased APAP-induced LDH released at concentrations of 5 and $10 \mu \mathrm{M}$ (Supplementary Figure 5A). It seems that montelukast has little effect on the expression of Cysltr1 (Supplementary Figure 5B). However, montelukast dramatically reversed APAP-induced mitochondrial membrane potential decreasing (Figure 5C). Zafirlukast, another kind of Cysltr1 antagonist, also improved APAP-induced hepatocyte death (Supplementary Figures 6A, B). Moreover, montelukast 
A Vehicle

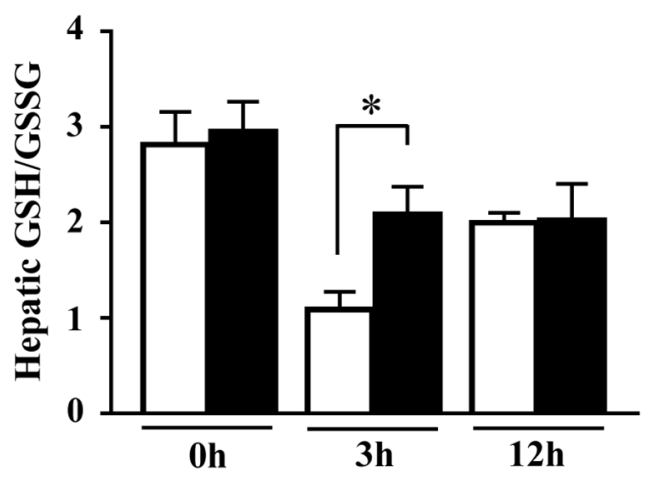

C

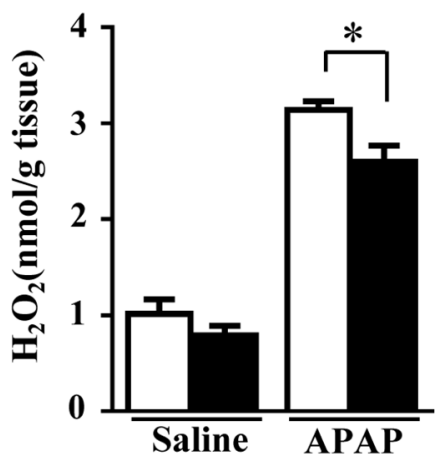

\section{B}

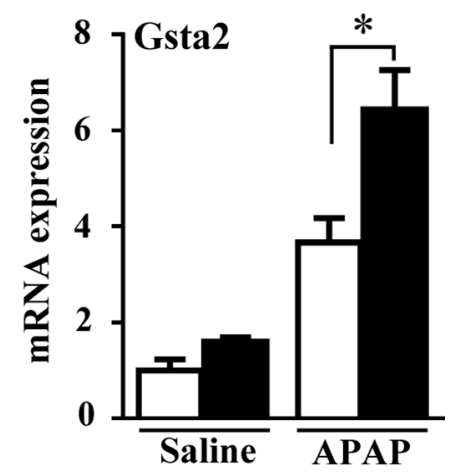

D

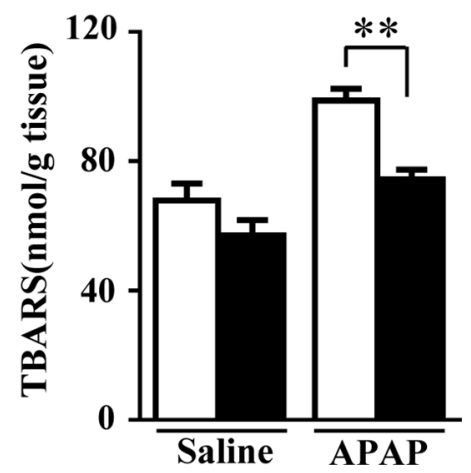

FIGURE 3 | Montelukast treatment maintained hepatic GSH level and reduced reactive oxygen species production in APAP treated mice. (A) Detection of hepatic glutathione (GSH)/glutathione disulfide (GSSG) level. (B) Real-time PCR analysis of hepatic mRNA expression of GSTa2. (C) Hepatic $\mathrm{H}_{2} \mathrm{O}_{2}$ level. (D) APAP-induced thiobarbituric acid reactive substances (TBARS) level. Data are mean \pm SEM, $n=5$ for saline groups, $n=7$ for APAP groups, ${ }^{\star} p<0.05,{ }^{\star \star} p<0.01$.

inhibited $\mathrm{LTD}_{4}$, a Cystlr1 agonist, induced hepatocytes damage (Supplementary Figure 7), and thus partly improved APAPinduced hepatotoxicity.

\section{DISCUSSION}

In this study, we showed that inhibition of Cysltr1 protected mice against APAP-induced liver damage. Montelukast postadministration inhibited APAP-induced hepatic oxidative stress and necrosis. Mechanistically, inhibition of Cysltr1 in APAP-treated mice induced the expression of GSTa2, which promotes NAPQI detoxification. Compared to the vehicle group, these protective effects were also associated with higher hepatic GSH/GSSG ratio and lower oxidative stress and JNK activity in montelukast-treated group. Montelukast administration also inhibited the inflammatory response with APAP overdose.

Phase II enzymes eliminate most APAP and protect livers from toxicity. The majority of APAP is metabolized by UGT and SULT, then excreted in the urine (McGill and Jaeschke, 2013).
Upregulated of UGT and SULT would be helpful to prevent toxicity of APAP overdosage (McGill and Jaeschke, 2013). However, montelukast did not alter the expression of Ugt1a1 and Sult $2 a 1$ in our study. Phase I metabolic enzymes and transporters also remained unchanged.

Our results showed that protective effect of montelukast in APAP-induced liver damage was associated with an increased level of hepatic GSH, which was thought to accumulate phase I metabolites detoxification. Glutathionylation is catalyzed by a group of enzymes called the GST. Gstm-null mice are found to be resistant to APAP (Arakawa et al., 2012). Upregulation of Gsta is also thought to prevent APAPinduced liver failure (Coles and Kadlubar, 2005; Gum and Cho, 2013). We also showed that montelukast increased GSTa2 expression after APAP administration, which may improve APAP-induced hepatotoxicity.

Recent studies have shown that montelukast has effects of antioxidant and anti-inflammation in various experimental models (Dengiz et al., 2007; Vollmar and Menger, 2011; Khodir et al., 2016). Pharmacological inhibition of Cysltr1 
A

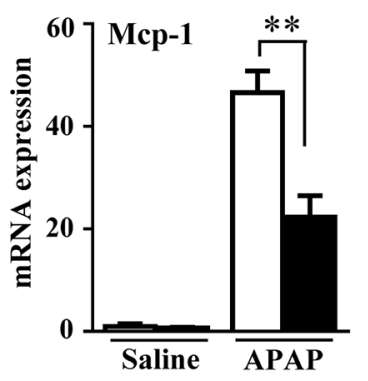

E

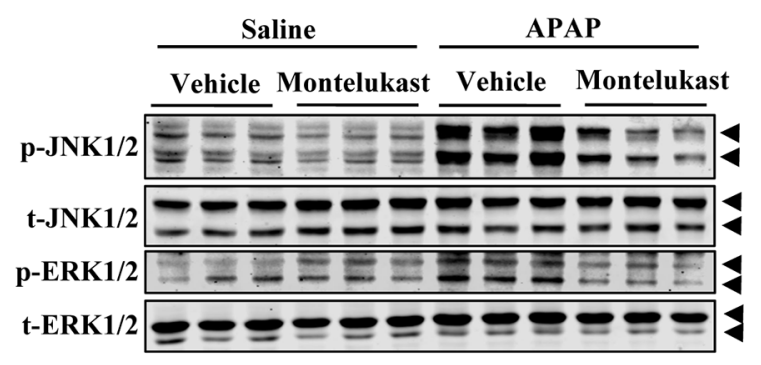

B

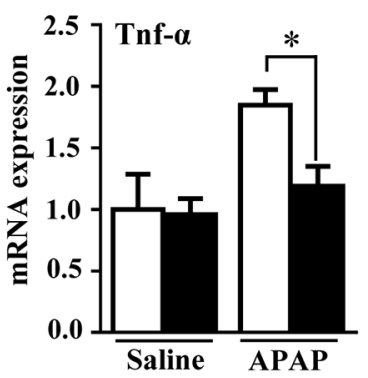

F

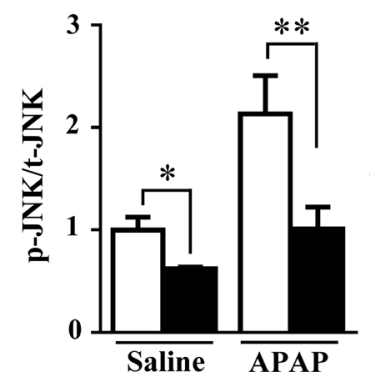

C

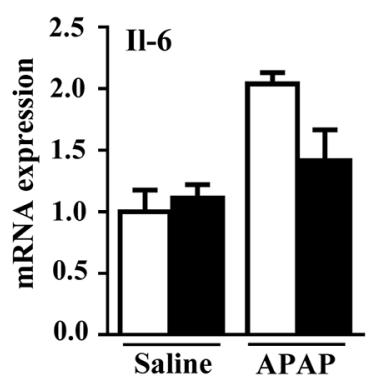

D

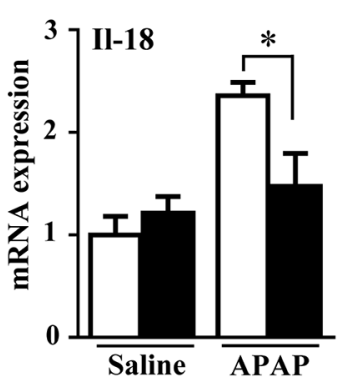

G

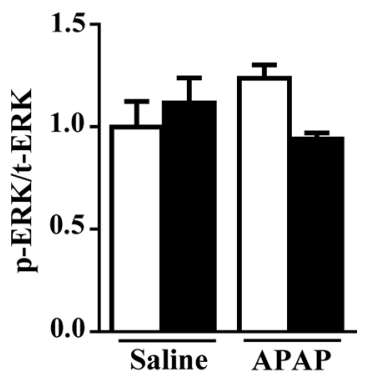

FIGURE 4 | Pharmacological inhibition of cysltr1 decrease APAP-induced hepatic inflammation. (A-D) Real-time PCR analysis of hepatic inflammatory cytokine genes expression, $n=5$ for saline groups, $n=7$ for APAP groups. (E) Western blotting analysis ERK and JNK phosphorylation. (F and $\mathbf{G})$ Quantification of phosphorylated JNK to total JNK and phosphorylated ERK to total ERK. Data are mean $\pm \mathrm{SEM}, n=3$ for each groups, ${ }^{\star} p<0.05,{ }^{* *} p<0.01$. Experiments were repeated three times with similar results.

protected aluminum-overload-induced liver injury through $\mathrm{PI} 3 \mathrm{~K} / \mathrm{AKT} / \mathrm{mTOR}$ pathway (Hu et al., 2018). At overdoses of APAP administration, hepatic GSH was depleted and APAP metabolites covalently bound to protein, which further exacerbates hepatic toxicity by triggering oxidative stress and JNK activation (Luster et al., 2000). Inhibition of Cysltr1 by montelukast treatment prevented APAP-induced oxidative stress and JNK activation. Activation of JNK mediates hepatocyte necrosis and trigger inflammation in APAP-induced liver injury model (Jaeschke et al., 2012; Woolbright and Jaeschke, 2017). Several studies suggested that inflammation plays a role in hepatocyte necrosis and is thought to promote liver injury (Hoque et al., 2012). The decreased activity of JNK and inflammation may contribute to protective effect of montelukast in APAP overdose model.

Studies have reported upregulation of 5-LO pathway in APAP-induced liver injury (Suciu et al., 2016). Activation of the 5-LO pathway leads to the formation of leukotrienes from arachidonic acid (Zhao et al., 2004). Leukotrienes are thought to be involved in the production of inflammation and lead to liver injury (Samuelsson et al., 1987; Kaminski, 1989; Martinez-Clemente et al., 2010). We also found $\mathrm{LTD}_{4}$-induced hepatocytes injury by promoting $\mathrm{LDH}$ released and decreasing mitochondrial membrane potential (Supplementary Figure 7). Knockout or inhibition of 5-LO could prevent APAP-induced liver inflammation and liver injury (Martinez-Clemente et al., 2010; Hohmann et al., 2013). Meanwhile, knockout or inhibition of leukotriene receptors is an effective way to treat tissue damage caused by leukotrienes (Beller et al., 2004). Several leukotriene receptors are identified. They are Blt1, Blt2, Cysltr1, Cysltr2, and Cysltr3. Functional consequences of Cysltr3 activation are not well defined (Evans et al., 2008). Although it has been reported that blocking Blt1 signaling protected APAP-induced hepatotoxicity through preventing excessive accumulation of hepatic neutrophils (Kojo et al., 2016), Blt1 receptor antagonists have no further development (Evans et al., 2008). Montelukast inhibited LTD $_{4}$-induced hepatocytes damage (Supplementary Figure 7) and thus partly improved APAP-induced hepatotoxicity. Therefore, montelukast administration may be a strategy for inhibiting APAP-induced liver injury.

It was attractive that APAP promoted Cysltr1 expression both in vivo and in vitro. The expression of Cysltr1 was significantly induced in the hepatic ischemia-reperfusion injury and aluminum-overload-induced liver injury (Wu et al., 2015; Hu et al., 2018). The elevated expression of Cysltr1 has been shown to participate the pathological progress of these diseases. In our study, we also found that Cysltr1 expression was higher in model of APAP-overdose-induced liver injury than the saline group. Blocking Cysltr1 by montelukast-alleviated APAP overdose 
A
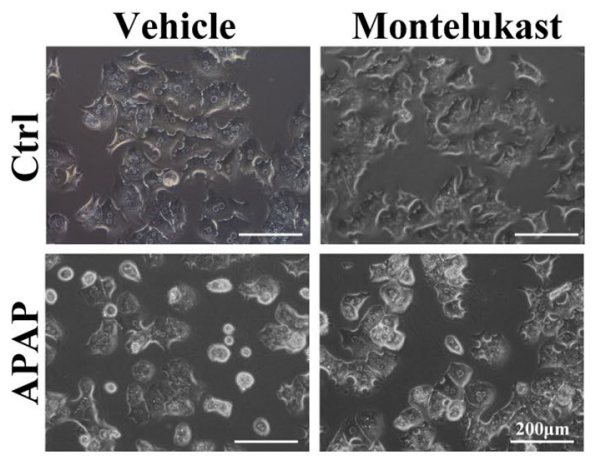

B

C

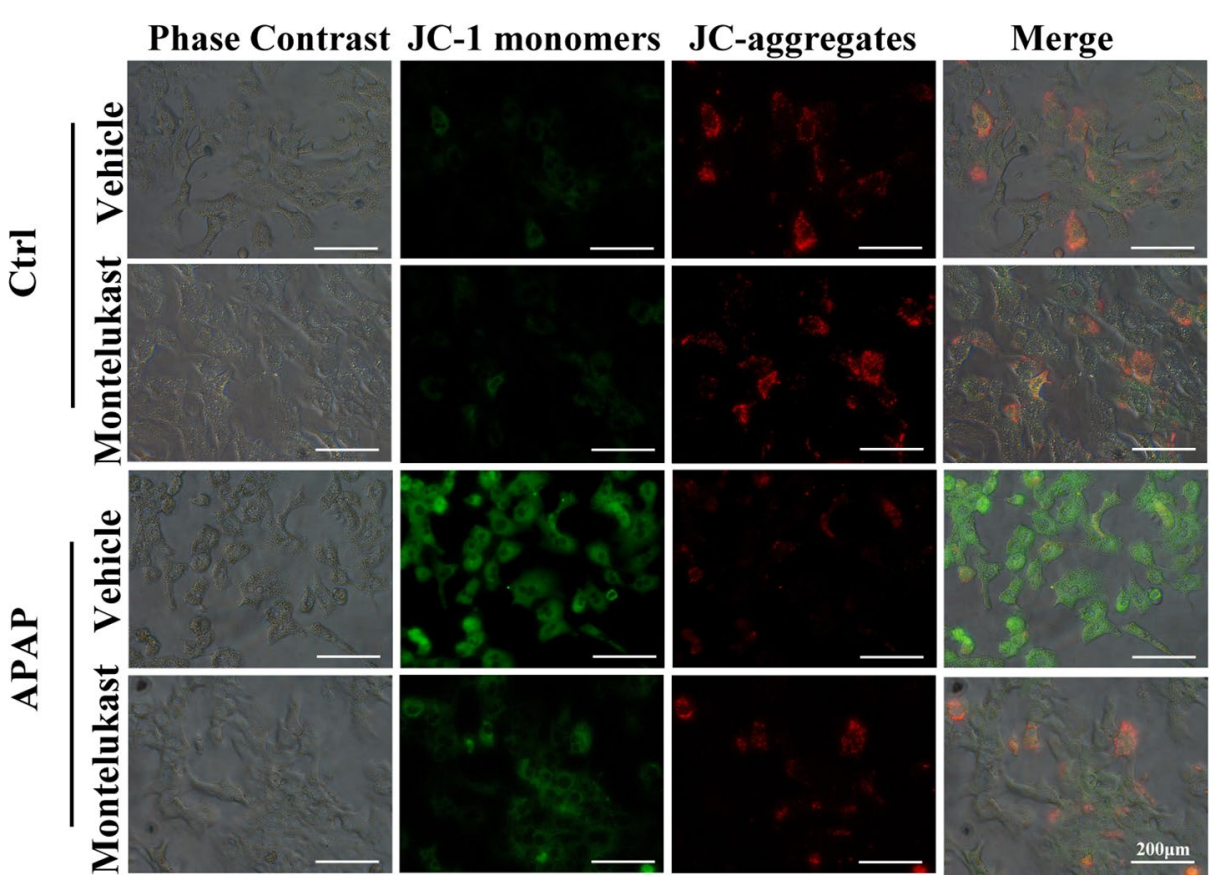

FIGURE 5 | Montelukast inhibit APAP-induced cell damage. Primary hepatocytes were pretreated with montelukast (5 $\mu$ M) or vehicle (DMSO) $1 \mathrm{~h}$ before APAP (2.5 $\mathrm{mM}$ ) administration. (A) Representative morphological images of primary hepatocytes treated with APAP for $24 \mathrm{~h}$. (B) Quantification of LDH released into the culture medium of primary hepatocyte after treatment with $2.5 \mathrm{mM}$ of APAP for $24 \mathrm{~h}$. Data are mean \pm SEM, $n=3$ for each group, ${ }^{* *} p<0.01$. (C) Primary hepatocytes were incubated with $5 \mathrm{mg} / \mathrm{l} \mathrm{JC}-1$ dye for $30 \mathrm{~min}$ at $37^{\circ} \mathrm{C}$ in the dark and washed twice with the dye buffer. Then, the cells were quickly subjected to a fluorescence microscope for captured red or green fluorescence. Experiments were repeated three times with similar results.

caused oxidative stress and inflammation. In vitro, it was reported that TNF- $\alpha$, an activator of necrosis, could stimulate Cysltr1 expression in human bronchi and thus promotes hyper-responsiveness (Khaddaj-Mallat et al., 2016). We also found that APAP upregulated Cysltr1 in primary hepatocytes, and it thereby enlarged hepatotoxicity. Future study is needed to study the mechanism of Cysltr1 upregulation under APAP overdose.

$\mathrm{N}$-acetylcysteine (NAC) is thought to be useful in the management of APAP-induced liver injury (Brok et al., 2006).
In APAP-treated liver, NAC is deacetylated to form L-cysteine, an essential amino acid for the synthesis of GSH (Smilkstein et al., 1991). One report compared the therapeutic effects of NAC and montelukast on APAP-induced hepatotoxicity and found that montelukast had better therapeutic effect than NAC did (Icer et al., 2016). However, they did not explain the mechanism of montelukast administration in APAPinduced hepatic damage. We observed an increased level of liver GSH/GSSG level, a reduced level of oxidative stress, JNK phosphorylation, and a decreased level of inflammation. 
These results will elucidate the protective mechanism of montelukast on APAP-induced liver injury. In the future, the prophylactic effect of montelukast should be further evaluated in APAP-induced liver damage.

In summary, the present study demonstrated that pharmacological inhibition of Cysltr1 by montelukast conferred resistance to APAP-induced hepatotoxicity. The protective effect of montelukast was, at least in part, attributed to upregulation of hepatic GSH/GSSG, suppression of oxidative stress and JNK pathway. Targeted inhibition of the Cysltr1 by montelukast may be a potential treatment strategy in APAPinduced hepatotoxicity.

\section{ETHICS STATEMENT}

This study was carried out in accordance with the recommendations of the guidelines of the animal care utilization committee of the institute, the Institutional Animal Care and Use Committee of Sichuan University. The protocol was approved by the Institutional Animal Care and Use Committee of Sichuan University.

\section{REFERENCES}

Arakawa, S., Maejima, T., Fujimoto, K., Yamaguchi, T., Yagi, M., Sugiura, T., et al. (2012). Resistance to acetaminophen-induced hepatotoxicity in glutathione S-transferase Mu 1-null mice. J. Toxicol. Sci. 37 (3), 595-605. doi: 10.2131/jts.37.595

Arthur, J. S., and Ley, S. C. (2013). Mitogen-activated protein kinases in innate immunity. Nat. Rev. Immunol. 13 (9), 679-692. doi: 10.1038/nri3495

Basavarajaiah, S., Sigston, P., and Budack, K. (2004). Severe salicylate poisoning treated conservatively. J. R. Soc. Med. 97 (12), 587-588. doi: 10.1258/jrsm.97.12.587

Beller, T. C., Friend, D. S., Maekawa, A., Lam, B. K., Austen, K. F., and Kanaoka, Y. (2004). Cysteinyl leukotriene 1 receptor controls the severity of chronic pulmonary inflammation and fibrosis. Proc. Natl. Acad. Sci. U. S. A. 101 (9), 3047-3052. doi: 10.1073/pnas.0400235101

Brok, J., Buckley, N., and Gluud, C. (2006). Interventions for paracetamol (acetaminophen) overdose. Cochrane Database Syst. Rev. 2, CD003328. doi: 10.1002/14651858.CD003328.pub2

Chen, L., Li, W., Qi, D., Lu, L., Zhang, Z., and Wang, D. (2018). Honokiol protects pulmonary microvascular endothelial barrier against lipopolysaccharideinduced ARDS partially via the Sirt3/AMPK signaling axis. Life Sci. 210, 86-95. doi: 10.1016/j.lfs.2018.08.064

Coles, B. F., and Kadlubar, F. F. (2005). Human alpha class glutathione S-transferases: genetic polymorphism, expression, and susceptibility to disease. Methods Enzymol. 401, 9-42. doi: 10.1016/S0076-6879(05)01002-5

Dengiz, G. O., Odabasoglu, F., Halici, Z., Suleyman, H., Cadirci, E., and Bayir, Y. (2007). Gastroprotective and antioxidant effects of amiodarone on indomethacin-induced gastric ulcers in rats. Arch. Pharm. Res. 30 (11), 14261434. doi: 10.1007/BF02977367

El-Boghdady, N. A., Abdeltawab, N. F., and Nooh, M. M. (2017). Resveratrol and montelukast alleviate paraquat-induced hepatic injury in mice: modulation of oxidative stress, inflammation, and apoptosis. Oxid. Med. Cell Longev. 2017, 9396425. doi: 10.1155/2017/9396425

Evans, J. F., Ferguson, A. D., Mosley, R. T., and Hutchinson, J. H. (2008). What's all the FLAP about?: 5-lipoxygenase-activating protein inhibitors for inflammatory diseases. Trends Pharmacol. Sci. 29 (2), 72-78. doi: 10.1016/j.tips.2007.11.006

Furuta, K., Yoshida, Y., Ogura, S., Kurahashi, T., Kizu, T., Maeda, S., et al. (2016). Gabl adaptor protein acts as a gatekeeper to balance hepatocyte death and proliferation during acetaminophen-induced liver injury in mice. Hepatology 63 (4), 1340-1355. doi: 10.1002/hep. 28410

\section{AUTHOR CONTRIBUTIONS}

SP and JH participated in research design. SP, QL, RL, ZZ, XY, and $\mathrm{CH}$ conducted experiments. SP, ZZ, and TW performed data analysis. SP, YL, and JH wrote or contributed to the writing of the manuscript.

\section{FUNDING}

This work was supported by the National Natural Science Foundation of China (81873662, 81870599, and 81603035), China Postdoctoral Fellowship (2017M612981), Young Scientist Fellowship of Sichuan University (2017SCU11026), and Postdoctoral Fellowship of Sichuan University (2017SCU12036).

\section{SUPPLEMENTARY MATERIAL}

The Supplementary Material for this article can be found online at: https://www.frontiersin.org/articles/10.3389/fphar.2019.01070/ full\#supplementary-material

Gao, W., Li, J., Li, Q., and An, S. (2018). CYSLTR1 promotes adenoid hypertrophy by activating ERK1/2. Exp. Ther. Med. 16 (2), 966-970. doi: 10.3892/ etm.2018.6282

Gum, S. I., and Cho, M. K. (2013). The amelioration of N-acetyl-p-benzoquinone imine toxicity by ginsenoside $\mathrm{Rg} 3$ : the role of Nrf2-mediated detoxification and Mrp1/Mrp3 transports. Oxid. Med. Cell Longev. 2013, 957947. doi: $10.1155 / 2013 / 957947$

Hamamoto, Y., Ano, S., Allard, B., O’Sullivan, M., McGovern, T. K., and Martin, J. G. (2017). Montelukast reduces inhaled chlorine triggered airway hyperresponsiveness and airway inflammation in the mouse. Br. J. Pharmacol. 174 (19), 3346-3358. doi: 10.1111/bph.13953

Hegab, I. I., El-Horany, H. E., Elbatsh, M. M., and Helal, D. S. (2018). Montelukast abrogates prednisolone-induced hepatic injury in rats: modulation of mitochondrial dysfunction, oxidative/nitrosative stress, and apoptosis. J. Biochem. Mol. Toxicol. 33, e22231. doi: 10.1002/jbt.22231

Hohmann, M. S., Cardoso, R. D., Pinho-Ribeiro, F. A., Crespigio, J., Cunha, T. M., Alves-Filho, J. C., et al. (2013). 5-Lipoxygenase deficiency reduces acetaminophen-induced hepatotoxicity and lethality. Biomed. Res. Int. 2013, 627046. doi: 10.1155/2013/627046

Hoque, R., Sohail, M. A., Salhanick, S., Malik, A. F., Ghani, A., Robson, S. C., et al. (2012). P2X7 receptor-mediated purinergic signaling promotes liver injury in acetaminophen hepatotoxicity in mice. Am. J. Physiol. Gastrointest Liver Physiol. 302 (10), G1171-G1179. doi: 10.1152/ajpgi.00352.2011

Hu, C., Yang, J., He, Q., Luo, Y., Chen, Z., Yang, L., et al. (2018). CysLTR1 blockage ameliorates liver injury caused by aluminum-overload via PI3K/AKT/mTORmediated autophagy activation in vivo and in vitro. Mol. Pharm. 15 (5), 19962006. doi: 10.1021/acs.molpharmaceut.8b00121

Icer, M., Zengin, Y., Gunduz, E., Dursun, R., Durgun, H. M., Turkcu, G., et al. (2016). Is montelukast as effective as $\mathrm{N}$-acetylcysteine in hepatic injury due to acetaminophen intoxication in rats? Exp. Toxicol. Pathol. 68 (1), 55-59. doi: 10.1016/j.etp. 2015.09 .008

Jaeschke, H., Williams, C. D., Ramachandran, A., and Bajt, M. L. (2012). Acetaminophen hepatotoxicity and repair: the role of sterile inflammation and innate immunity. Liver Int. 32 (1), 8-20. doi: 10.1111/j.1478-3231.2011.02501.x

Kaminski, D. L. (1989). Arachidonic acid metabolites in hepatobiliary physiology and disease. Gastroenterology 97 (3), 781-792. doi: 10.1016/0016-5085(89)90655-0

Khaddaj-Mallat, R., Sirois, C., Sirois, M., Rizcallah, E., Marouan, S., Morin, C., et al. (2016). Pro-resolving effects of resolvin D2 in LTD4 and TNF-alpha 
pre-treated human bronchi. PLoS One 11 (12), e0167058. doi: 10.1371/journal. pone. 0167058

Khodir, A. E., Ghoneim, H. A., Rahim, M. A., and Suddek, G. M. (2016). Montelukast attenuates lipopolysaccharide-induced cardiac injury in rats. Hum. Exp. Toxicol. 35 (4), 388-397. doi: 10.1177/0960327115591372

Kizu, T., Yoshida, Y., Furuta, K., Ogura, S., Egawa, M., Chatani, N., et al. (2015). Loss of Gabl adaptor protein in hepatocytes aggravates experimental liver fibrosis in mice. Am. J. Physiol. Gastrointest Liver Physiol. 308 (7), G613-G624. doi: 10.1152/ajpgi.00289.2014

Kojo, K., Ito, Y., Eshima, K., Nishizawa, N., Ohkubo, H., Yokomizo, T., et al. (2016). BLT1 signalling protects the liver against acetaminophen hepatotoxicity by preventing excessive accumulation of hepatic neutrophils. Sci. Rep. 6, 29650. doi: $10.1038 /$ srep29650

Kondeti, V., Al-Azzam, N., Duah, E., Thodeti, C. K., Boyce, J. A., and Paruchuri, S. (2016). Leukotriene D4 and prostaglandin E2 signals synergize and potentiate vascular inflammation in a mast cell-dependent manner through cysteinyl leukotriene receptor 1 and E-prostanoid receptor 3. J. Allergy Clin. Immunol. 137 (1), 289-298. doi: 10.1016/j.jaci.2015.06.030

Lee, W. M. (2017). Acetaminophen (APAP) hepatotoxicity-Isn't it time for APAP to go away? J. Hepatol. 67 (6), 1324-1331. doi: 10.1016/j.jhep.2017.07.005

Lei, C., Wu, S., Wen, C., Li, Y., Liu, N., Huang, J., et al. (2019). Zafirlukast attenuates advanced glycation end-products (AGEs)-induced degradation of articular extracellular matrix (ECM). Int. Immunopharmacol. 68, 68-73. doi: 10.1016/j. intimp.2018.12.056

Luster, M. I., Simeonova, P. P., Gallucci, R. M., Matheson, J. M., and Yucesoy, B. (2000). Immunotoxicology: role of inflammation in chemical-induced hepatotoxicity. Int. J. Immunopharmacol. 22 (12), 1143-1147. doi: 10.1016/ S0192-0561(00)00073-4

Lynch, K. R., O’Neill, G. P., Liu, Q., Im, D. S., Sawyer, N., Metters, K. M., et al. (1999). Characterization of the human cysteinyl leukotriene CysLT1 receptor. Nature 399 (6738), 789-793. doi: 10.1038/21658

Martinez-Clemente, M., Ferre, N., Gonzalez-Periz, A., Lopez-Parra, M., Horrillo, R., Titos, E., et al. (2010). 5-Lipoxygenase deficiency reduces hepatic inflammation and tumor necrosis factor alpha-induced hepatocyte damage in hyperlipidemia-prone ApoE-null mice. Hepatology 51 (3), 817-827. doi: 10.1002/hep. 23463

McGill, M. R., and Jaeschke, H. (2013). Metabolism and disposition of acetaminophen: recent advances in relation to hepatotoxicity and diagnosis. Pharm. Res. 30 (9), 2174-2187. doi: 10.1007/s11095-013-1007-6

McGovern, T., Goldberger, M., Chen, M., Allard, B., Hamamoto, Y., Kanaoka, Y., et al. (2016). CysLT1 receptor is protective against oxidative stress in a model of irritant-induced asthma. J. Immunol. 197 (1), 266-277. doi: 10.4049/ jimmunol.1501084

Nakagawa, H., Maeda, S., Hikiba, Y., Ohmae, T., Shibata, W., Yanai, A., et al. (2008). Deletion of apoptosis signal-regulating kinase 1 attenuates acetaminopheninduced liver injury by inhibiting c-Jun $\mathrm{N}$-terminal kinase activation. Gastroenterology 135 (4), 1311-1321. doi: 10.1053/j.gastro.2008.07.006

Osman, J., Savari, S., Chandrashekar, N. K., Bellamkonda, K., Douglas, D., and Sjolander, A. (2017). Cysteinyl leukotriene receptor 1 facilitates tumorigenesis in a mouse model of colitis-associated colon cancer. Oncotarget 8 (21), 3477334786. doi: 10.18632/oncotarget. 16718
Pu, S., Ren, L., Liu, Q., Kuang, J., Shen, J., Cheng, S., et al. (2016). Loss of 5-lipoxygenase activity protects mice against paracetamol-induced liver toxicity. Br. J. Pharmacol. 173 (1), 66-76. doi: 10.1111/bph.13336

Saini, S. P., Zhang, B., Niu, Y., Jiang, M., Gao, J., Zhai, Y., et al. (2011). Activation of liver $\mathrm{X}$ receptor increases acetaminophen clearance and prevents its toxicity in mice. Hepatology 54 (6), 2208-2217. doi: 10.1002/hep.24646

Samuelsson, B., Dahlen, S. E., Lindgren, J. A., Rouzer, C. A., and Serhan, C. N. (1987). Leukotrienes and lipoxins: structures, biosynthesis, and biological effects. Science 237 (4819), 1171-1176. doi: 10.1126/science.2820055

Shayiq, R. M., Roberts, D. W., Rothstein, K., Snawder, J. E., Benson, W., Ma, X., et al. (1999). Repeat exposure to incremental doses of acetaminophen provides protection against acetaminophen-induced lethality in mice: an explanation for high acetaminophen dosage in humans without hepatic injury. Hepatology 29 (2), 451-463. doi: 10.1002/hep.510290241

Smilkstein, M. J., Bronstein, A. C., Linden, C., Augenstein, W. L., Kulig, K. W., and Rumack, B. H. (1991). Acetaminophen overdose: a 48-hour intravenous $\mathrm{N}$-acetylcysteine treatment protocol. Ann. Emerg. Med. 20 (10), 1058-1063. doi: 10.1016/S0196-0644(05)81352-6

Suciu, M., Gruia, A. T., Nica, D. V., Azghadi, S. M., Mic, A. A., and Mic, F. A. (2016). Data on expression of lipoxygenases-5 and - 12 in the normal and acetaminophendamaged liver. Data Brief 7, 1199-1203. doi: 10.1016/j.dib.2016.03.079

Thummel, K. E., Lee, C. A., Kunze, K. L., Nelson, S. D., and Slattery, J. T. (1993). Oxidation of acetaminophen to N-acetyl-p-aminobenzoquinone imine by human CYP3A4. Biochem. Pharmacol. 45 (8), 1563-1569. doi: 10.1016/0006-2952(93)90295-8

Vollmar, B., and Menger, M. D. (2011). Intestinal ischemia/reperfusion: microcirculatory pathology and functional consequences. Langenbecks Arch. Surg. 396 (1), 13-29. doi: 10.1007/s00423-010-0727-x

Woolbright, B. L., and Jaeschke, H. (2017). Role of the inflammasome in acetaminophen-induced liver injury and acute liver failure. J. Hepatol. 66 (4), 836-848. doi: 10.1016/j.jhep.2016.11.017

Wu, S., Zhu, X., Jin, Z., Tong, X., Zhu, L., Hong, X., et al. (2015). The protective role of montelukast against intestinal ischemia-reperfusion injury in rats. Sci. Rep. 5, 15787. doi: 10.1038/srep 15787

Yan, M., Huo, Y., Yin, S., and Hu, H. (2018). Mechanisms of acetaminopheninduced liver injury and its implications for therapeutic interventions. Redox Biol. 17, 274-283. doi: 10.1016/j.redox.2018.04.019

Zhao, L., Moos, M. P., Grabner, R., Pedrono, F., Fan, J., Kaiser, B., et al. (2004). The 5-lipoxygenase pathway promotes pathogenesis of hyperlipidemia-dependent aortic aneurysm. Nat. Med. 10 (9), 966-973. doi: 10.1038/nm1099

Conflict of Interest Statement: The authors declare that the research was conducted in the absence of any commercial or financial relationships that could be construed as a potential conflict of interest.

Copyright $\odot 2019 \mathrm{Pu}, \mathrm{Liu}, \mathrm{Li}, \mathrm{Li}$, Wu, Zhang, Huang, Yang and He. This is an open-access article distributed under the terms of the Creative Commons Attribution License (CC BY). The use, distribution or reproduction in other forums is permitted, provided the original author(s) and the copyright owner(s) are credited and that the original publication in this journal is cited, in accordance with accepted academic practice. No use, distribution or reproduction is permitted which does not comply with these terms. 